\title{
Criterios para la configuración de plataformas de inteligencia aumentada para el mejoramiento de la sostenibilidad de cultivos agrícolas
}

\author{
Alejandro Peña-Palacio \\ pfjapena@gmail.com / Grupo de Investigación \\ en Inteligencia Computacional y Automática de la Universidad EIA \\ (Medellín, Colombia)
}

Recepción: 31/10/2020 Aceptación: 4/11/2020

RESUMEN. El desarrollo de la inteligencia artificial ha planteado una serie de retos en cuanto al futuro y la sostenibilidad del trabajo humano. Sin embargo, el desarrollo de la tecnología ha traído consigo el desarrollo de conceptos como el de la inteligencia aumentada (IA), el cual tiene por objetivo el mejoramiento de las capacidades humanas mediante la interacción hombre-máquina para la solución de problemas complejos en diferentes áreas del conocimiento. Esta interacción supone una serie de retos desde lo tecnológico, ya que la experiencia humana es un proceso complejo de transfer learning para que las máquinas sean un complemento perfecto de las personas. En el contexto de la agricultura de precisión, las plataformas de inteligencia aumentada (AIP, por sus siglas en inglés) han surgido como una alternativa importante para el fortalecimiento de las capacidades en la detección y diagnóstico de estados fitosanitarios o agroclimáticos. En este artículo, se propone una metodología para la configuración de las AIP, integrando tres elementos que son fundamentales para la sostenibilidad de cultivos como son: imágenes áreas espectrales utilizando vehículos aéreos no tripulados (UAV, por sus siglas en inglés), mapas de pronóstico para describir la dispersión de enfermedades y sus vectores asociados en campo, modelos deep y machine learning para la caracterización automática de eventos fitosanitarios o agroclimáticos, así como redes IoT-IoB (Internet of Things e Internet of Beings) para la interacción hombre-dispositivos. Para la evaluación de estas plataformas, se propone un GAP de sostenibilidad, el cual evalúa de una manera integral la reducción en el uso de pesticidas y fertilizantes, así como la sostenibilidad de los puestos de trabajo en un futuro de largo plazo, y en donde la inteligencia artificial tendrá un papel preponderante en el desarrollo agrícola en el mundo.

Palabras Clave: inteligencia aumentada / imágenes multiespectrales / machine y deep learning / redes IoT-IoB / riesgo operacional 


\title{
Criteria for the Configuration of Augmented Intelligence Platforms for Improving Agricultural Crop Sustainability
}

\begin{abstract}
The development of artificial intelligence has posed several challenges regarding the future and sustainability of human work. However, the development of technology has brought concepts such as augmented intelligence (AI), which aims to improve human capabilities through human-machine interaction to solve complex problems in different areas of knowledge. This interaction entails a series of technological challenges since the human experience is a complex transfer learning process in which machines are a perfect complement to people. In the context of precision agriculture, AI platforms (AIPs) have emerged as an important alternative to strengthen capacities for the detection and diagnosis of phytosanitary or agroclimatic conditions. This article proposes a methodology for the configuration of AIPs by integrating three fundamental elements for the sustainability of crops: spectral aerial images using unmanned aerial vehicles (UAVs), forecast maps to describe the spread of diseases and their associated vectors in the field, deep \& machine learning models for the automatic characterization of phytosanitary or agroclimatic events, and IoT-IoB (Internet of Things \& Internet of Beings) networks for human-device interaction. For the evaluation of these platforms, a sustainability gap is proposed, which comprehensively assesses the reduction in pesticide and fertilizer use, as well as the sustainability of jobs in the long-term future where artificial intelligence will play a leading role in the agricultural development in the world.
\end{abstract}

KEYWORDS: augmented intelligence / multispectral image / machine \& deep learnin / IoT-IoB networks / operational risk 


\section{INTRODUCCIÓN}

El desarrollo de la inteligencia artificial a nivel mundial ha planteado una serie de retos en cuanto al futuro del trabajo y de las personas que lo desarrollan (Oppenheimer, 2018). En este contexto, muchos investigadores han manifestado la preocupación por la aparición de las máquinas inteligentes como sustitutas de las personas; sin embargo, otros investigadores han apelado al desarrollo de la inteligencia artificial como sistemas para el soporte a la toma de decisiones, mientras que otra tendencia de desarrollo (Zhao, Dong y Ying, 2020) se centra en el paradigma de la inteligencia aumentada, en donde la inteligencia artificial tiene un papel preponderante con el fin de aumentar las capacidades humanas para la solución de problemas complejos, manteniendo un balance entre las personas y la tecnología (Talaviya, Shah, Patel, Yagnik y Shah, 2020).

En el contexto de la agricultura de precisión, uno de los retos para mejorar la sostenibilidad de cultivos agrícolas se centra en la identificación de riesgos asociados con eventos de tipo fitosanitario y agroclimático. Esta identificación se ha hecho de manera ancestral por personas que tienen cierta experiencia en la identificación de estos eventos para diferentes cultivos, la cual cada vez escasea más en el mundo. Sin embargo, la seguridad alimentaria del planeta ha hecho que estos cultivos se desarrollen en grandes extensiones de tierra, lo cual supone una limitante para la experiencia humana (Shirsath, Vyas, Aggraval y Kolli, 2019).

Para mejorar la sostenibilidad ambiental y financiera de cultivos agrícolas en general, muchos investigadores han apelado al uso de diferentes tecnologías entre las que se destacan las imágenes espectrales y satelitales (Fitrianto, Darmawan, Tokimatsu y Sufwandika, 2018), los vehículos aéreos no tripulados (UAV, por sus siglas en inglés) para la fumigación y fertilización automática de cultivos (Baseca, Sendra, Lloret y Tomas, 2019), y el diseño de redes IoT-IoB (Internet of Things e Internet of Beings) para la interacción entre dispositivos y equipos (Popovic, Nedeljko, Pesic, Zecevic, Krstajic y Djukanovik, 2017), modelos Lagrangian Machine Learning, modelos para el modelamiento de la dispersión de contaminantes y de enfermedades en campo (Peña, Hernández y Toro, 2010), así como modelos borrosos para la evaluación de riesgo operacional (Peña et al., 2018). Estas tecnologías han mostrado, a lo largo de los años, desarrollos completamente independientes, lo cual nos permite evaluar claramente la participación humana en estas tendencias como lo establece la inteligencia aumentada (Talaviya et al., 2020), así como la evaluación de parámetros de riesgo en cultivos agrícolas afectados por un evento fitosanitario en el contexto FinTech-InsurTech (Shirsath et al., 2019). Estas tendencias de desarrollo marcan los elementos fundamentales para la construcción de plataformas de inteligencia aumentada destinadas al mejoramiento de la sostenibilidad ambiental y financiera como se propone en la metodología.

Para la solución del problema del mejoramiento de la sostenibilidad ambiental y financiera de cultivos agrícolas, en este documento se proponen una serie de criterios para el desarrollo de plataformas de inteligencia aumentada que permitan superar las limitantes impuestas por 
el desarrollo de cultivos en grandes extensiones de terreno afectadas por eventos fitosanitarios. Es importante mencionar que para el caso de cultivos agrícolas estas plataformas utilizarán como centro del proceso el uso de imágenes áreas de baja altura, ya sean de tipo multiespectral (IMA) o hiperespectral (IHA) utilizando vehículos aéreos no tripulados (UAV), el desarrollo de modelos computacionales lagrangianos soportados en la inteligencia artificial para describir la dispersión de enfermedades en tierra, así como redes IoT-IoB para la interacción entre dispositivos y humanos para la solución de problemas complejos, tal y como lo supone la agricultura de precisión. En un contexto de sostenibilidad, estas AIP son evaluadas teniendo en cuenta herramientas financieras que den viabilidad a la integración de tecnología, la disminución en el uso de pesticidas y fertilizantes, manteniendo en todo momento la participación humana (Peña et al., 2018; Castrignano, Buttafuoco, Khosla, Mouazen, Moshou y Naud, 2020).

La estructura de este artículo está definida por tres secciones. Una primera sección o de introducción, en donde se hace una identificación de las principales tendencias de desarrollo en esta área del conocimiento. Una segunda sección en donde se describen los índices de vegetación (IV) y la estructura de datos, los elementos que componen una AIP, así como la definición del GAP de sostenibilidad, que busca medir el desempeño de un modelo en un contexto de sostenibilidad. Finalmente, una tercera sección muestra las conclusiones y recomendaciones que establecen los criterios para el mejoramiento de la sostenibilidad ambiental y financiera de cultivos agrícolas en general, utilizando las AIP.

\section{METODOLOGÍA}

En el contexto de la agricultura de precisión, la interacción autónoma entre dispositivos y equipos con el fin de aumentar las capacidades humanas en el mejoramiento de la sostenibilidad ambiental y financiera de cultivos agrícolas, como lo sugiere la inteligencia aumentada, supone todo un reto desde lo tecnológico. Para dar solución a este reto, se propone la siguiente metodología.

\section{1 Índices de vegetación y estructura de datos}

La palma de aceite es un cultivo oleaginoso que se ha extendido en el mundo gracias a su alto potencial productivo (Lai, Tan y Akoh, 2015). Se estima que una hectárea produce entre seis a diez veces más aceite que otro tipo de cultivos similares y se ubica levemente por debajo de la eficiencia del aceite de soya (Corley y Tinker, 2015). A pesar de la presencia que tiene la palma de aceite en el sector de los alimentos, por ser una fuente natural enriquecida de ácido palmítico, su cultivo no ha estado exento de polémicas, ya que este se ha desarrollado en zonas de gran reserva ecológica a nivel mundial, y porque se ha visto afectado por enfermedades como la marchitez letal (ML), la pudrición de cogollo (PC) o la ganoderma, lo que supone el uso de grandes cantidades de pesticidas y fertilizantes (Kathun, Reza, Moniruzzaman y Yaakob, 2017). 
Para el análisis de la sostenibilidad ambiental y financiera de cultivos agrícolas en el contexto de la agricultura de precisión, las metodologías de análisis de riesgo sugieren tomar como referencia un total de 1000 unidades de cultivo (144 unidades-7 ha) en general (Peña et al., 2018). Para la captura de unidades de cultivo, se utilizó un vehículo aéreo no tripulado de la serie DJI Phantom 3 (https://www.dji.com/phantom-3-pro) equipado con una cámara multiespectral de la serie Sequoia Parrot (https://www.parrot.com/en/shop/accessories-spareparts/other-drones/sequoia), la cual permite la toma de imágenes multiespectrales a diferentes alturas y a cuatro bandas de frecuencia: Green $(550 \mathrm{~nm} / 40 \mathrm{~nm}), \operatorname{Red}(660 \mathrm{~nm} / 40 \mathrm{~nm})$, Red Edge $(735 \mathrm{~nm} / 40 \mathrm{~nm})$, Near Infrarred $(790 \mathrm{~nm} / 40 \mathrm{~nm})$, a una resolución de $13 \mathrm{~cm} /$ px y a una altura máxima de $120 \mathrm{~m}$ (MAI).

De acuerdo con la dispersión de la ML en cultivos de palma de aceite, estas unidades de cultivo fueron agrupadas en tres categorías de acuerdo con la metodología propuesta por Giraldo (2016): palmas sanas fuera de foco (SFF), palmas aparentemente sanas dentro de foco (A-SDF) y palmas con marchitez letal evidente (ML) (figura 1).

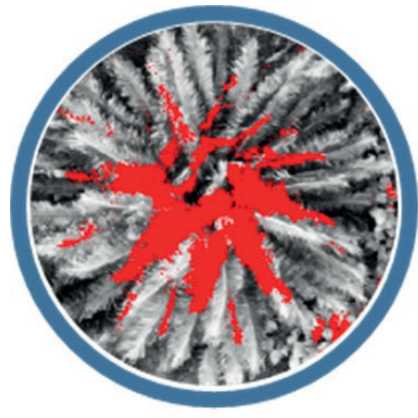

(a)

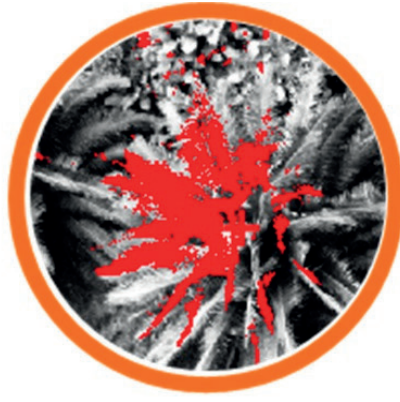

(b)

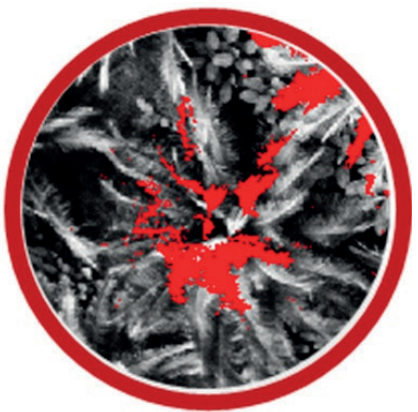

(c)

Figura 1. Unidades de cultivo de palma y su respuesta espectral en el infrarrojo cercano (NIR). (a) Palma SFF.

(b) Palma A-SDF. (c) Palma ML.

Elaboración propia

Para evaluar el vigor vegetal utilizando imágenes áreas multiespectrales (IMA), la agricultura de precisión apela a los índices de vegetación, entre los que se destacan el índice NDV, el GNDVI y el RNVI como los definen Díaz (2015) y Peña, Bonet, Manzur, Góngora y Carffini (2019). En la figura 2, se puede observar la estructura característica de un índice de vegetación (GNDVI) para una imagen multiespectral tomada a una altura de 10 metros. 


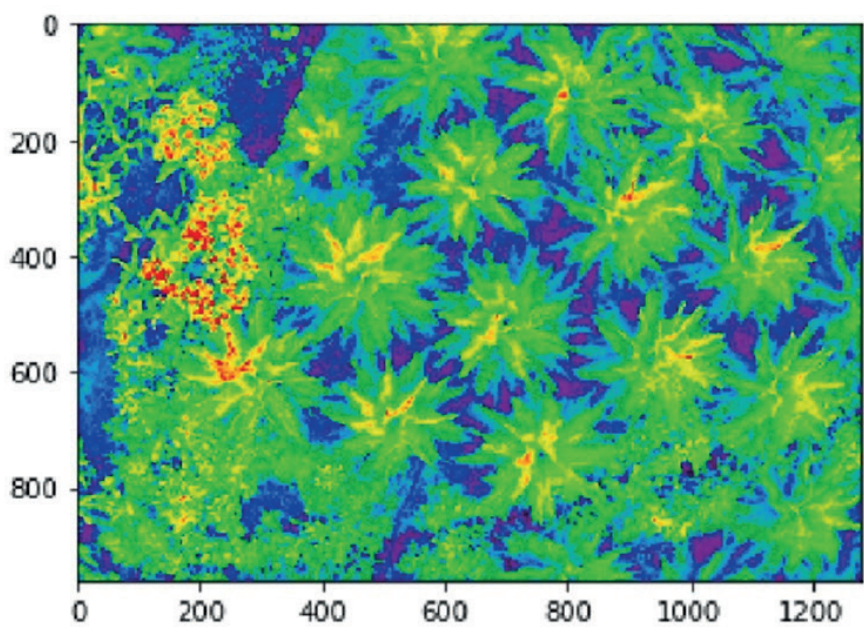

(a)
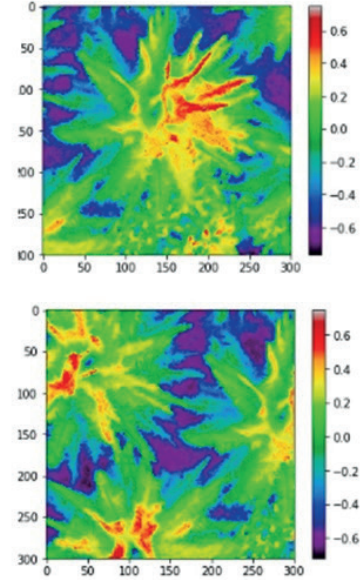

(b)

Figura 2. Índices de vegetación. (a) Unidades de cultivo morfológicamente completas. (b) Unidades de cultivo morfológicamente incompletas.

Elaboracion propia

La metodología general para la evaluación de la sostenibilidad ambiental y financiera de este tipo de plataformas se enmarca dentro del análisis del riesgo de las operaciones del negocio de una organización según los Acuerdos de Basilea (Trenca, Pece y Sorina, 2015; Peña et al., 2018). De esta manera, un escenario de referencia para la evaluación estará configurado de la siguiente manera: SFF: 600 uds., SDF: 100 uds., ML: 300 uds. (Molina, Acosta, Torres y Hernández, 2019).

\subsection{Estructura de una plataforma de inteligencia aumentada}

De manera general, una plataforma de inteligencia aumentada, destinada al mejoramiento de la sostenibilidad de cultivos agrícolas, está compuesta por tres elementos fundamentales que se describen a continuación, y como se muestran en la figura 3 (Popovic et al., 2017): 


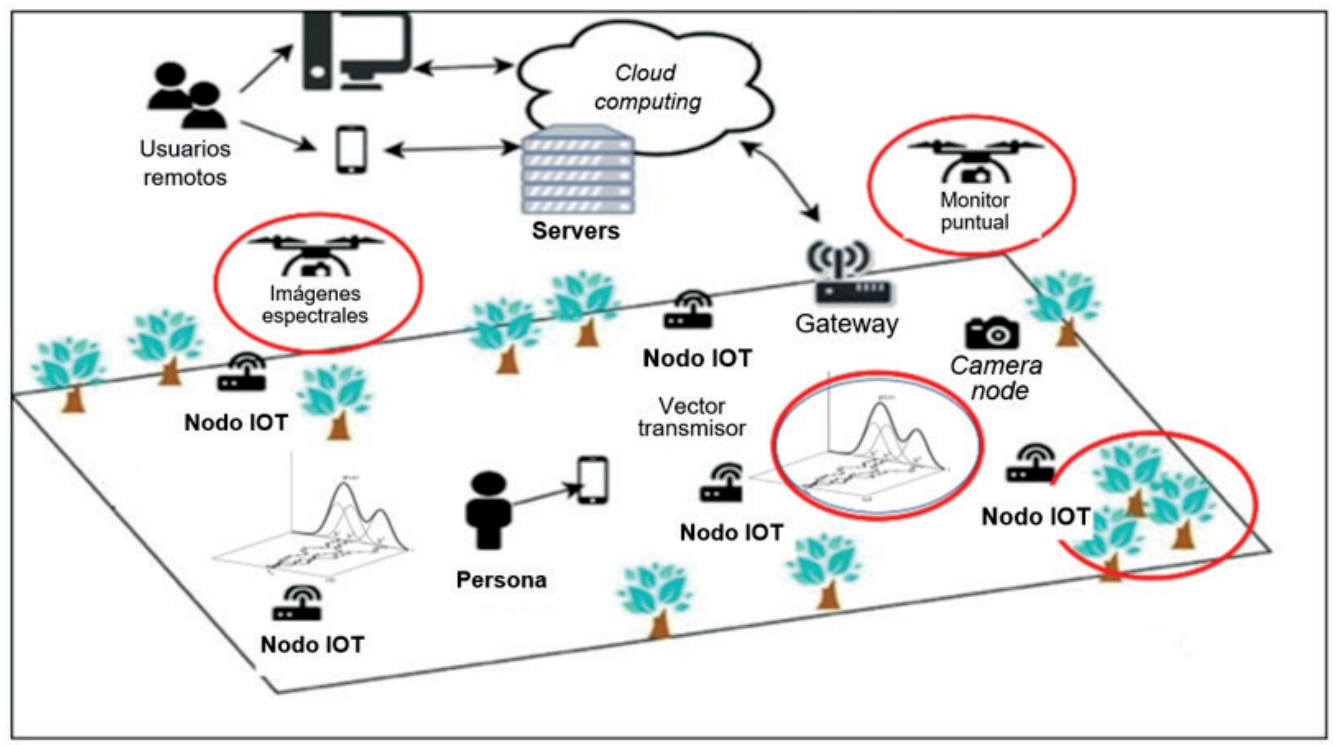

Figura 3. Estructura general de plataformas de inteligencia aumentada en cultivos agrícolas

Fuente: Popovic et al. (2017)

\subsection{Mapas de pronóstico (patrones de dispersión)}

Los mapas de pronóstico, a diferencia de los índices de vegetación, articulan en una sola estructura la dinámica de la evolución de un estado fitosanitario, integrando conceptos de dinámica lagrangiana (Peña, Hernández y Toro, 2010). Estos mapas de pronóstico poseen dos objetivos fundamentales. Un objetivo para la caracterización temporal de unidades de cultivo de palma y su evolución temporal en las categorías que define la ML (ML, A-SDF, SFF); y un segundo objetivo, el cual permite la diferenciación con otro tipo de patologías de dispersión como la que genera la PC o la ganoderma. Desde el punto de vista del machine learning, este tipo de estructuras o patrones de dispersión permite extender la autonomía de los UAV por efecto de una captura localizada de las IMA en grandes extensiones de terreno, como lo sugiere este tipo de cultivos. En la figura 4 se puede observar la evolución de la ML por unidad de cultivo. 


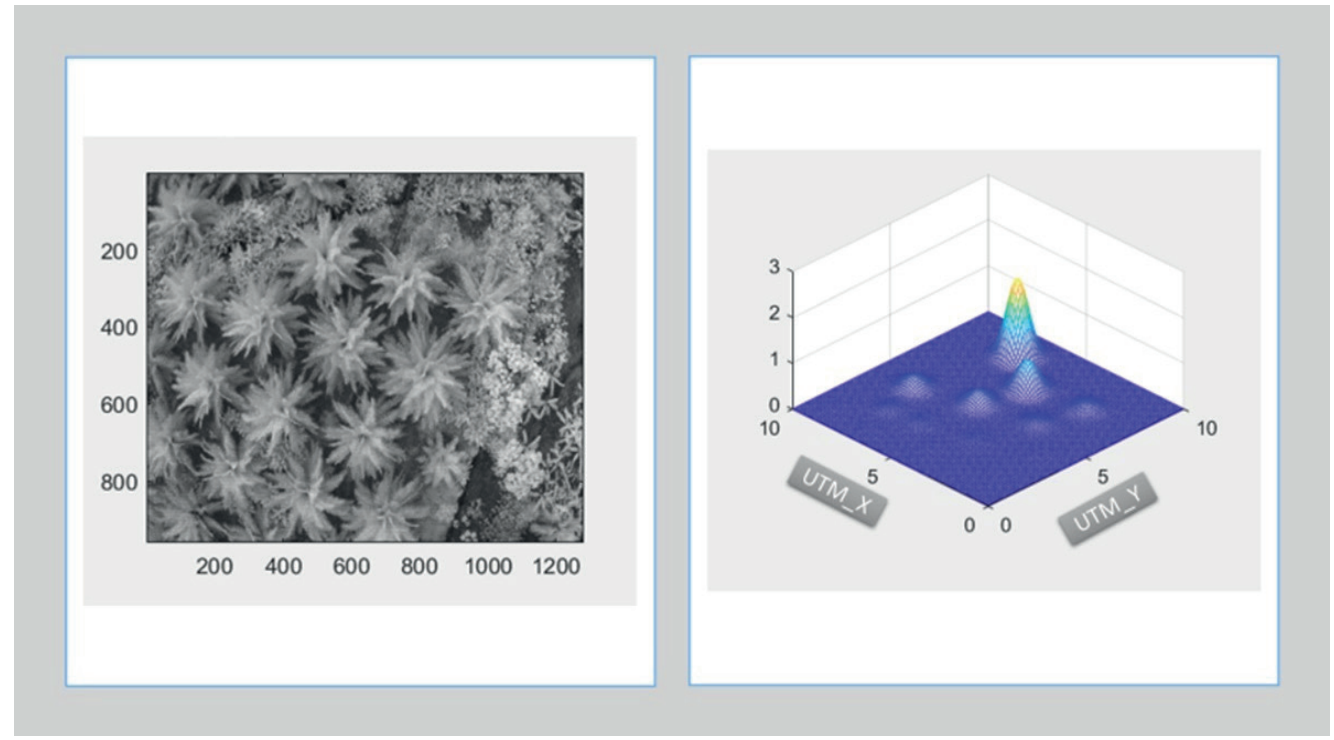

(a)

(b)

Figura 4. Mapa de pronóstico para la dispersión de la marchitez letal (ML). (a) Imagen multiespectral.

(b) Mapa de pronóstico.

Elaboración propia

En la figura 4 se pueden observar diferentes unidades de cultivo con diferentes niveles de afectación por ML. Aquí, las campanas gaussianas más esbeltas muestran la presencia de unidades de cultivo de palma con síntomas evidentes de ML y en donde el monitoreo en vuelo se debe hacer con una mayor frecuencia, mientras que campanas más extendidas dan lugar a palmas en las categorías SFF y A-SDF.

\subsection{Modelos deep learning}

Dentro del contexto de las plataformas de inteligencia aumentada, los modelos machine y deep learning soportan el proceso de transfer learning con el fin de mejorar las capacidades humanas en el diagnóstico y la detección de unidades de cultivo de palma afectadas por ML tomando como referencia los mapas de pronóstico descritos anteriormente. La estructura general para un modelo con estructura stacked deep learning se denota y define:

$$
y r_{k}=\operatorname{softmax}\left(\sum_{j=1}^{N O} C_{j, k}\left(\sum_{j_{3}=1}^{N O_{3}} w_{j_{3}, j_{2}}\left(\sum_{j_{2}=1}^{N O_{2}} w_{j_{2}, j_{1}}\left(\sum_{j_{1}=1}^{N O_{1}} w_{j_{1}, j_{o}} \cdot x_{j_{o}, k}\right)\right)\right)\right)
$$


Donde:

$y r_{k}$ : define la función softmax que define las categorías de clasificación de unidades de cultivo por categorías de afectación (ML, A-SDF, SFF).

$x_{0, k}:$ representa cada uno de los puntos de control que conforman un índice de vegetación dinámico para un instante de tiempo $k$.

$C_{j_{m}, j_{n}}, w_{j_{l} j_{o}}$ : representan los soportes de la estructura para el modelo. Los pesos $w_{j_{l}, j_{o}}$ son obtenidos utilizando estrategias de autoencoder (Liu, Bao y Baokun, 2018), mientras que los pesos que soportan la estructura de la capa de salida $\left(C_{j_{m}, j_{n}}\right)$ son obtenidos mediante la utilización de la regla delta generalizada (Mohanty, Hughes y Salathé, 2016).

\subsection{Redes tipo IoT-IoB}

Las plataformas de inteligencia aumentada permiten no solo la interacción entre dispositivos y equipos, sino que permiten también la interacción entre humanos y dispositivos. Para tal efecto, estas plataformas precisan de nodos IoT (Internet of Things) para la medición puntual de parámetros y variables en campo (e.g., humedad, acidez del suelo, dispersión de enfermedades y contaminantes, censos foliares en campo utilizando IMA). Esta información es enviada a través de protocolos de comunicación y API, en donde los nodos IoT se comunican de forma directa e indirecta a través de un gateway con servidores en la nube (cloud computing) como se muestra en la figura 3 (Popovic et al., 2017).

La integración de los dos elementos anteriores (e.g., mapas de pronóstico, modelos deep learning) a la estructura de comunicación de la plataforma dará lugar al concepto de Internet of Beings (IoB), el cual tiene por objeto integrar a los datos IoT la dinámica de un fenómeno físico para el mejoramiento de las prestaciones de dispositivos y equipos, y que para el caso particular de cultivos agrícolas, este concepto está determinado por dinámicas de dispersión de contaminantes y enfermedades en campo, así como para mejorar la autonomía de los UAV destinados a la captura de las IMA (Castrignano et al., 2020).

\subsection{GAP de sostenibilidad (GS)}

El análisis de plataformas AIP supone un análisis tecnológico-financiero en el contexto del riesgo operacional (Peña et al., 2018). Desde el punto de vista agrícola, este análisis se basa en la caracterización de parámetros de riesgo a partir de la detección y diagnóstico de eventos fitosanitarios (Molina, Acosta, Torres y Hernández, 2019), la reducción en el uso de pesticidas y fertilizantes (Talaviya, Shah, Patel, Yagnik y Shah, 2020), así como desde la eficiencia de la experiencia humana en campo (Giraldo, 2016).

En el contexto de la agricultura de precisión, los riesgos operacionales se pueden clasificar de la siguiente manera: categoría C1 (PC1), en donde se agrupan las pérdidas esperadas, las cuales 
están asociadas al monitoreo y control de plagas, así como al mantenimiento, la poda y la fertilización de los cultivos. Por su parte, los riesgos categoría C2 (PC2) agrupan todas las pérdidas no esperadas por imprevistos en el manejo del cultivo o por eventos agroclimáticos inesperados. Finalmente, los riesgos categoría C3 (PC3) se conocen como riesgos catastróficos, y surgen por efecto de un mal tratamiento del cultivo frente a un evento de tipo fitosanitario.

La caracterización de los riesgos en plataformas AIP está soportada por los modelos transfer y deep learning, y se relacionan con el buen desempeño de dichos modelos en la detección y diagnóstico de la ML de acuerdo con el caso de estudio propuesto. Aquí, los riesgos C1 se asocian con los costos de operación, diagnóstico y detección de la ML en sus tres categorías de afectación (SFA, A-SDF, ML); los riesgos C2, con los costos de la detección y diagnóstico de la ML en campo por la falta de especificidad de la plataforma; mientras que los riesgos categoría C3 agrupan todos los costos asociados a la falta de sensibilidad del modelo en la detección de palmas A-SDF, lo que puede llevar a la erradicación errónea de unidades de cultivo. Estos riesgos se conocen como riesgos catastróficos. Para el caso particular de cultivos de palma afectados por ML, el diferencial de sostenibilidad $\left(D S_{\mathrm{n}}\right)$ se denota y define:

$$
D S_{\mathrm{n}}=P C 1-P C 3
$$

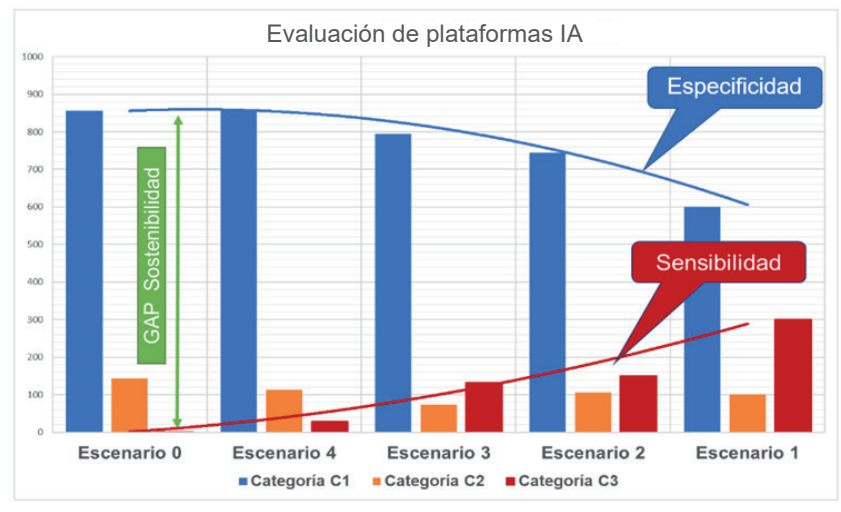

Figura 5. Análisis técnico-financiero de plataformas de IA

Elaboración propia

En la figura 5 se pueden observar tres escenarios de desempeño teórico de la AIP en la detección y diagnóstico de la ML en campo (escenario 2, escenario 3, escenario 4), tomando como referencia la configuración de la muestra de unidades de cultivo definidas para este estudio (SFF: 600 uds., A-SDF: 400 uds., ML: 100 uds.). De esta manera, el GAP de sostenibilidad (GS) se puede evaluar en términos de dos escenarios de referencia, un escenario en el cual no existe la intervención humana por efecto de la falta del recurso con la experiencia en campo (escenario 1), y un escenario de referencia (escenario 0) que reúne los criterios de 
sostenibilidad RSPO (RSPO, 2018). De esta manera, el GAP de sostenibilidad (GS) como resultado del desempeño de una AIP para su mejor configuración (escenario $n$ ) se denota y define:

$$
G S=D S_{0}-D S_{\mathrm{n}}
$$

El GAP de sostenibilidad da lugar a la configuración de parámetros de riesgo para la cobertura diferenciada de estos activos (e. g., PC1, PC2, PC3), y se alinea con el desarrollo InsurTech, el cual tiene por objetivo el desarrollo de seguros paramétricos (Kath, Mushtag, Henry, Adewuyi y Stone, 2018).

En la figura 5 y en la tabla 1 se puede observar cómo el $D S_{\mathrm{n}}$ se va ampliando a medida que se genera una mejor gestión del cultivo. Esto trae consigo un GAP financiero más amplio, por efecto de una reducción natural en el uso de pesticidas y fertilizantes, y por efecto de una mayor eficiencia en la detección y diagnóstico de la ML, garantizando de esta manera la sostenibilidad de los puestos de trabajo que pueden ser reemplazados por el ingreso de la tecnología, todo esto en consonancia con los postulados que establecen las normas RSPO a nivel mundial en esta materia (RSPO, 2018).

Tabla 1

Escenarios de riesgo-GAP de sostenibilidad

\begin{tabular}{|c|c|c|c|c|c|}
\hline Escenarios & Escenario 0 & Escenario 1 & Escenario 3 & Escenario 4 & Escenario 1 \\
\hline Nivel & RSPO & $\begin{array}{c}\text { Gestión } \\
\text { proactiva }\end{array}$ & $\begin{array}{c}\text { Gestión } \\
\text { preventiva }\end{array}$ & $\begin{array}{l}\text { Gestión } \\
\text { reactiva }\end{array}$ & $\begin{array}{c}\text { Evolución } \\
\text { natural }\end{array}$ \\
\hline $\begin{array}{l}\text { Hectáreas } \\
\text { (unidades) }\end{array}$ & 6,9 ha (1000 uds.) & 6,9 ha (1000 uds.) & 6,9 ha (1000 uds.) & 6,9 ha (1000 uds.) & 6,9 ha (1000 uds.) \\
\hline $\begin{array}{c}N D \\
E I-U L-(S L)\end{array}$ & $856-143-(1)$ & $832-89-(79)$ & $818-109-(73)$ & $762-87-(151)$ & $600-100-(300)$ \\
\hline PC3 (USD) & $\$ 259,03$ & $\$ 9932,54$ & $\$ 32751,93$ & $\$ 45918,31$ & $\$ 96579,88$ \\
\hline PC3 (USD) & $\$ 6691,71$ & $\$ 3612,09$ & $\$ 2834,91$ & $\$ 3898,07$ & $\$ 4163,86$ \\
\hline PC3 (USD) & $\$ 7881,24$ & $\$ 7739,87$ & $\$ 5897,37$ & $\$ 4739,97$ & $\$ 3885,04$ \\
\hline$D S_{n}$ & 855 & 753 & 745 & 611 & 300 \\
\hline GS & $\$ 92324,64$ & $\$ 82792,51$ & $\$ 61815,62$ & $\$ 49806,64$ & - \\
\hline
\end{tabular}

\section{CONCLUSIONES}

Las AIP se constituyen en una alternativa tecnológica para mejorar la sostenibilidad ambiental y financiera no solo de cultivos agrícolas, sino de las operaciones del negocio de una organización en donde la experiencia humana juega un papel determinante. Para el caso particular de cultivos agrícolas, estas plataformas permitirán el fortalecimiento y la eficiencia de las 
capacidades humanas en el diagnóstico de eventos fitosanitarios y agroclimáticos en cultivos agrícolas que cubren grandes extensiones de terreno.

Gracias a su capacidad de adaptación y aprendizaje, las AIP pueden ser extendidas fácilmente para la detección y diagnóstico de estados fitosanitarios en otro tipo de cultivos en donde se requiere la identificación de unidades de cultivo morfológicamente completas en una zona de estudio. Sin embargo, el éxito de este tipo de plataformas no está en la identificación de unidades de cultivo independientes, sino en la identificación de patrones de dispersión como los definen los mapas de pronóstico, lo que va mucho más allá del uso de índices de vegetación.

Las AIP se constituyen en un elemento fundamental para el desarrollo FinTech (artificial intelligence financial products) e InsurTech (artificial intelligence insurance products) a nivel mundial en el contexto agrícola, ya que la integración de modelos y dispositivos permite la configuración de parámetros de riesgo que dan lugar a la estructura de seguros paramétricos, o para la configuración de coberturas diferenciadas para la protección de activos, cumpliendo con uno de los postulados de estas tendencias, que es el mejoramiento de la sostenibilidad ambiental y financiera de cualquier organización.

Uno de los retos que afronta la inteligencia aumentada desde la interacción hombre máquina está centrado principalmente en las metodologías transfer learning, las cuales requerirán de la creación de métodos formales para la captura de la experiencia humana por parte de un sistema computacional. Para el sector agrícola, la interacción hombre-máquina llevará a una caracterización eficiente de eventos fitosanitarios y agroclimáticos en etapa temprana, por fortalecimiento de las capacidades humanas en campo, lo que impactará positivamente en la sostenibilidad ambiental y financiera para este tipo de actividades. Para el caso de cultivos de palma de aceite, esta sostenibilidad se verá reflejada en el corto plazo en una reducción en el uso de pesticidas y fertilizantes, alcanzando los objetivos de sostenibilidad que establecen las normas RSPO a nivel mundial y que pueden ser extendidos fácilmente para otro tipo de cultivos agrícolas.

\section{REFERENCIAS}

Aleaur, S. (s. f.). Building a Sustainable Future Together! https://www.sulitest.org

Anderson, R. (Director). (2009). The Business Logic of Sustainability [Película].

Anthropocene. (24 de enero del 2021). En Wikipedia. https://en.wikipedia.org/wiki/ Anthropocene

Baseca, C., Sendra, S., Lloret, J., y Tomas, J. (2019). A Smart Decision System for Digital Farming. Agronomy, 9(5). https://10.3390/agronomy9050216 
Broman, G. I., y Robèrt, K.-H. (2017). A framework for strategic sustainable development. Journal of Cleaner Production, 140, 17-31.

Castrignano, A., Buttafuoco, G., Khosla, R., Mouazen, A., Moshou, D., y Naud, O. (2020). Agricultural Internet of Things and Decision Support for Precision Smart Farming. Elsevier. doi: ISBN 9780128183731

Corley, R. H. V., y Tinker, B. (2015). The Oil Palm (Fifth edition). Wiley Online Library.

Díaz, J. (2015). Estudio de indices de vegetación a partir de imágenes aéreas tomadas desde UAS/ RPAS y aplicaciones de estos a la agricultura de precisión. Universidad Complutense de Madrid.

Fitrianto, A., Darmawan, A., Tokimatsu, K., y Sufwandika, M. (2018). Estimating the Age of Oil Palm Trees Using Remote Sensing Technique. IOP Conference Series: Earth and Environmental Science, 148, 012020. https://10.1088/1755-1315/148/1/012020

Giraldo, R. (2016). Estudio de firmas espectrales de palmas de aceite afectadas con la marchitez letal, usando análisis estadísticos de datos funcionales. Palmas, 37, 131-139. https:// publicaciones.fedepalma.org/index.php/palmas/article/view/11897

Global Footprint Network. (s. f.). Earth Overshoot Day. https://www.footprintnetwork.org/ our-work/earth-overshoot-day/

Kath, J., Mushtag, S., Henry, R., Adewuyi, A., y Stone, R. (2018). Index Insurance Benefits Agricultural Producers Exposed to Excessive Rainfall Risk. Weather and Climate Extremes, 22, 1149-1159. https://doi.org/10.1016/j.wace.2018.10.003

Kathun, R., Reza, M., Moniruzzaman, M., y Yaakob, Z. (2017). Sustainable Oil Palm Industry: The Possibilities. Renewable and Sustainable Energy Reviews, 76, 608-619. https:// doi.org/10.1016/j.rser.2017.03.077

Lai, O. M., Tan, C. P., y Akoh, C. C. (2015).Palm Oil: Production, Processing, Characterization, and Uses. Elsevier Science. https://doi.org/10.1016/C2015-0-02411-X

Liu, G., Bao, H., y Baokun, H. (2018). A Stacked Autoencoder-Based Deep Neural Network for Achieving Gearbox Fault Diagnosis. Mathematical Problems in Engineering, 1-10. https://10.1155/2018/5105709

Max-Neef, M., Elizalde, A., y Hopenhayn, M. (1991). Human Scale Development: Conception, Application and Further Reflections. Zed Books.

Mohanty, S., Hughes, D., y Salathé, M. (2016). Using Deep Learning for Image-Based Plant Disease Detection. Frontiers in Plant Science, 7, 1419-1429. https://10.3389/ fpls.2016.01419 
Molina, V., Acosta, M., Torres, J., y Hernández, J. (Octubre del 2019). Diferencias entre el comportamiento espectral de palmas sanas y palmas afectadas por marchitez letal (ML). ResearchGate. https://www.researchgate.net/publication/336197147_Diferencias_ entre_el_comportamiento_espectral_de_palmas_sanas_y_palmas_afectadas_por_ Marchitez_letal_ML

Oppenheimer, A. (2018). ;Sálvese quien pueda! El futuro del trabajo en la era de la automatización. Debate.

Peña, A., Bonet, I., Lochmuller, C., Patiño, H., Chiclana, F., y Góngora, M. (2018). A Fuzzy Credibility Model to Estimate the Operational Value at Risk Using Internal and External Data of Risk Events. Knowledge-Based Systems, 159, 98-109. https://doi. org/10.1016/j.knosys.2018.06.007

Peña, A., Bonet, I., Manzur, D., Góngora, M., y Carffini, F. (2019). Validation of Convolutional Layers in Deep Learning Models to Identify Patterns in Multispectral Images. Proceedings of 14th. Conference on Informations Systems and Technologies (pp. 1-6). Systems Information and Technologies Association (AISTI). https://doi. org/10.23919/CISTI.2019.8760741

Peña, A., Bonet, I., Lochmuller, C., Chiclana, F., y Góngora, M. (2018). Flexible Inverse Adaptive Fuzzy Inference Model to Identify the Evolution of Operational Value at Risk for Improving Operational Risk Management. Applied Soft Computing, 65, 614-631. https://doi.org/10.1016/j.asoc.2018.01.024

Peña, A., Hernández, J., y Toro, M. (2010). Computational Evolutionary Inverse Lagrangian Puff Model. Environmental Modelling y Software, 25(12), 1890-1893. http://www. sciencedirect.com/science/article/pii/S1364815210001192

Popovic, T., Nedeljko, L., Pesic, A., Zecevic, Z., Krstajic, B., y Djukanovik, S. (2017). Architecting an IoT-Enabled Platform for Precision Agriculture and Ecological Monitoring: A Case Study. Computers and Electronics in Agriculture, 140, 255-265. https://doi.org/10.1016/j.compag.2017.06.008

Richard, R., y Lundholm, K. (2005). Engaging Individuals to Act Strategically Towards Sustainability. School of Engineering Blekinge Institute of Technology.

RSPO. (2013). RSPO Principles and Criteria for the Production of Sustainable Palm Oil. http://www.rspo.org/key-documents/certification/rspo-principles-and-criteria

Shirsath, P., Vyas, S., Aggraval, P., y Kolli, R. (2019). Designing Weather Index Insurance of Crops for the Increased Satisfaction of Farmers, Industry and the Government. Climate Risk Management, 25. https://doi.org/10.1016/j.crm.2019.100189 
Sivers, D. (Director). (2010). How to start a movement [Película].

Steffen, W., Richardson, K., Rockström, J., Cornell, S. E., Fetzer, I., Bennett, E. M., Biggs, R., Carpenter, S. R., de Vries, W., de Wit, C. A., Folke, C., Gerten, D., Heinke, J., Mace, G. M., Persson, L. M., Ramanathan, V., Reyers, B., y Sörlin, S. (2015). Planetary boundaries: Guiding human development on a changing planet. Science, 347(6223). https://doi.org/10.1126/science.1259855

Sulitest. (2020). Raising y Mapping Awareness of the Global Goals. United Nations virtual edition. https://www.sulitest.org/hlpf2020report.pdf.

Talaviya, T., Shah, D., Patel, N., Yagnik, H., y Shah, M. (2020). Implementation of Artificial Intelligence in Agriculture for Optimisation of Irrigation and Application of Pesticides and Herbicides. Artificial Intelligence in Agriculture, 4, 58-73. https://doi. org/10.1016/j.aiia.2020.04.002

Trenca, I., Pece, A. M., y Mihuț, I. S. (2015). The Assessment of Market Risk in the Context of the Current Financial Crisis. Procedia Economics and Finance, 32, 1391-1406. https://doi.org/10.1016/S2212-5671(15)01516-6

United Nations Department of Economic and Social Affairs. (s. f.). The 17 Goals Sustainable Development. https://sdgs.un.org/goals

World Commission on Environment and Development. (1987). Our Common Future. Oxford University Press.

Zhao, S., Dong, Y., y Ying, H. (2020). The Reliability Analysis of Rating Systems in Decision Making: When Scale Meets Multi-Attribute Additive Value Model. Decision Support Systems, 138. https://doi.org/10.1016/j.dss.2020.113384 